INTERNATIONAL DESIGN CONFERENCE - DESIGN 2018

https://doi.org/10.21278/idc.2018.0486

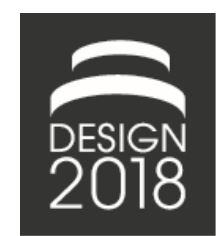

\title{
CONCEPTUAL FRAMEWORK FOR ANALYSING KNOWLEDGE DYNAMICS IN ENGINEERING SCIENCE
}

\author{
A. Märten, J. F. W. Jenek, W. M. Wang, C. Fleck, H. Meyer, R. Stark and S. Ammon
}

\begin{abstract}
Despite increasing knowledge intensity in product development, there is still a lack of understanding regarding the dynamic interrelations of knowledge types, stakeholders and artefacts in value creation processes.This dilemma counts also for engineering science as a discipline. This paper proposes a conceptual framework that allows for the analysis of dynamic knowledge flows in practical design processes and fundamental engineering research. Results from exemplary applications on two use cases will be used to illustrate its overall applicability and its potentials for science and industry.
\end{abstract}

Keywords: knowledge dynamics, design knowledge, biologically inspired, case study, engineering design

\section{Introduction}

Engineering science represents an academic field incorporating a wide range of disciplines. Characteristic for engineering science is its practice-oriented nature; and, hence, research in this field is often allocated at the intersection between fundamental research and practical application. Respective projects aim at solving specific technical problems or at developing functional technical systems by drawing on theoretical knowledge of various disciplines from within and outside of engineering sciences. The creation of new technical or technological solutions or the realization of epistemic advances demands acquisition and transformation of existing knowledge on the one hand and generation and integration of new knowledge in the ongoing developmental processes on the other. The degree of abstraction of the research object can vary considerably within engineering science. On strongly application-oriented level, the development of technical products can be regarded as engineeringscientific research. Engineers apply and combine their knowledge to create artefacts, which evolve in iterative cycles and finally result in a technical product (Neumann, 2015). Due to the increasing complexity of modern products, a deep understanding of how knowledge is processed in such development processes is crucial for industrial companies to survive in global competition. On a product level, the knowledge intensity in product development is mainly driven by heterogeneous market and technology trends as well as by the convergence of physical products with digital services. On the process level, the increasing number of software components and service elements requires close cooperation between different disciplines over the entire product life cycle, thus broadening the horizon of actual product development (Müller et al., 2016). The situation is similar in the field of research where the increasing complexity of research topics necessitates of large interdisciplinary research projects, which bring together knowledge from diverse scientific disciplines (Wang et al., 2016).

Similar to the diversity of research objects in engineering science, there are also various understandings and definitions of the term "knowledge". Within the scientific and epistemic tradition, knowledge is 
usually defined as "true and justified belief" (von Kutschera, 1982). It describes something that is approved by society and has stabilized through processes of observation and experiment. Within the engineering tradition, the definition of the term knowledge often draws more on the technical terms of data and information. North (2011) describes data as a basic resource for information and knowledge. According to him, data per se are merely collections of symbols that need a specific context in which it can be interpreted to gain a meaning and to become information. By further linking of information, e.g. for a specific purpose, knowledge emerges, which enables individuals to solve problems or to make decisions. However, given the current state of research, there is a lack of understanding concerning the dynamics of knowledge in relation to processes and phenomena within the broader network of disciplines in engineering science. In order to meet this challenge, the joint research project "Knowledge dynamics in engineering sciences" addresses this challenge by investigating how knowledge is dealt with systematically in engineering science. Our interdisciplinary team of researchers examines from different perspectives how knowledge is handled in different situations and at different levels of observation. We wish to develop a generic framework for describing and analyzing the dynamics of knowledge in processes of engineering science. The core of this framework comprises six key concepts that characterize different aspects of processing and manifesting knowledge. We claim that the flow and development of knowledge in engineering science projects can be made visible and comprehensible by applying this framework.

In order to explore the applicability of this conceptual framework, we will draw our investigation on two case studies, which derive from different disciplines within the engineering sciences. The project "Development of a fabric alignment device" (FAD) comes from the field of mechanical engineering and describes an application-oriented product development project. The project "Bioinspired Materials" is located in the field of materials science and describes fundamental research as part of a collaborative research project. These two projects were chosen as examples because they are located at opposite poles of a research spectrum, which ranges from an application-oriented research aiming at developing a unique product to foundational research aiming at generalizable insights and laws. Thus, both projects offer a good opportunity to examine the applicability of the proposed conceptual framework.

In the following section, the key concepts of the framework are introduced. Section 3 describes both case studies in detail to allow a better understanding. Whereas Section 4 applies the framework and its key concepts on the case studies, the results are discussed in Section 5. Finally, Section 6 presents conclusions, which derive from the application of the framework, as well as an outlook for further research.

\section{Conceptual framework and key concepts}

The successful development of modern products strongly depends on the steady exchange of knowledge between the involved stakeholders (Gausemeier and Wiendahl, 2011). Design engineers find it increasingly difficult to cope with the ever-growing amount of data, information and heterogeneous knowledge. Consequently, they have to spend significant amounts of time for coordination and communication tasks as well as for identification and gathering of information (Lünnemann et al., 2016). Hence, knowledge management (KM) plays an essential role in engineering processes. In order to support engineers in their daily work, different approaches, such as expert systems or knowledge-based engineering methods (cf. Verhagen et al., 2012), have been developed in order to capture and preserve specific engineering knowledge and to enable its further utilization. Moreover, guidelines such as VDI5610 from the Association of German Engineers (Verband Deutscher Ingenieure, VDI) have been developed to provide methodological guidance for implementing KM in product development. In this guideline, five core activities are described: 1) Planning, identifying and evaluating knowledge; 2) Creating knowledge; 3) Store Knowledge; 4) Distribution of knowledge; 5) Application of knowledge (VDI, 2008). However, this guideline is primarily based on the technical understanding of knowledge (cf. North (2011) as described in Section 1) and has a narrow focus on processes of product development. Thus, the concept cannot be applied easily to more fundamental engineering scientific research. Furthermore, the VDI5610 is not designed to generate a profound understanding of knowledge-related activities, but rather to provide practical guidelines to integrate $\mathrm{KM}$ in product development processes. Therefore, it fosters an in-depth understanding of the development processes itself, but does not focus on the causal interrelations between kinds of knowledge, involved human actors and artifacts. The conceptual framework presented here will contribute to close that gap by providing a tool to analyze the complex 
interrelations between activities, artefacts and human actors in development processes, both in science and engineering. It is based on six key concepts of knowledge dynamics, which are able to capture knowledge activities in the engineering sciences in broader terms. We claim that these generic terms allow us to describe core activities in knowledge processing and to capture the dynamic "flow" of knowledge.

The first term knowledge acquisition is defined as targeted acquisition and preparation of knowledge regarding a specific problem using different techniques, resources and media. It refers to activities such as the research for and the gathering of existing knowledge resources in order to utilize them for the given problem.

Knowledge transformation describes the conversion and further development of selected (external) knowledge stocks in order to make them applicable for a context in question. By this, modifications, translations, simplifications or aggregation take place.

Knowledge generation summarizes activities for creating new knowledge by addressing engineeringscientific issues. This term may relate to theoretical approaches, e.g. theory formation, and practical activities such as testing and prototyping.

Knowledge integration describes all activities of (systematic) coordination of available knowledge stocks as well as their merging into envisioned results, e.g. semi-finished or finished products and processes.

Knowledge transfer means the distribution of knowledge derived from technical and engineeringscientific development processes. Among other things, the aspects of transfer path, medium, direction, actors, interfaces and content are considered within this term.

The sixth term knowledge manifestation covers all kinds of artefacts that are created, used or manipulated in processes of engineering research. Such artefacts manifest the knowledge applied by individuals within engineering processes. Theoretically, individuals distinct from those who created this knowledge can make this knowledge (or parts of it) accessible again through interpretation. The term knowledge manifestation differs from the previous ones by describing static artefacts rather than dynamic activities. Nonetheless, the concept is essential for the framework, since it allows to capture multiplication and circulation of knowledge beyond the mode of direct interaction between humans.

It is worth mentioning that this framework should not be understood as a process model. This impression may arise at first glance, as the described concepts may suggest a logical and temporal order. In fact, the framework serves as an analytical model in order to examine the dynamics of knowledge in different phases of engineering research.

\section{Description of the case studies}

\subsection{Case study 1: Development of a fabric alignment device}

The case study refers to the project „Infinitus“- the world's largest digital printer for printing on soft materials. It was funded from 2010 to 2012 by the Central Innovation Program for Medium-Sized Businesses (Zentrales Innovationsprogramm Mittelstand) of the German Federal Ministry of Economic Affairs and Energy. Three researchers and three students with a background in mechanical and information engineering, at the Chair Machinery System Design (MSD), TU Berlin, collaborated with Big Image Systems Deutschland GmbH (BI) in order to plan, develop, construct and actually build a user-friendly, ecologically beneficial and stable endless-printing technology for flexible materials up to a width of twelve meter applicable to stages, decorations and events. Two sub-projects were involved in "Infinitus": the development of a fabric alignment device to prepare fabrics before introducing them to the printing system and the development of a guide rail and drive for an exactly adjustable high-speed printer head. In our case study, we will draw on the former sub-project.

At the beginning of the project, two main problems were identified. Firstly, there was the challenge of the length of printing products. Existing printing technologies already enabled the printing on twelvemeter wide materials, which is actually the maximum woven size, but the length of each tissue was limited to the perimeter length of the BI's printing drum. Bigger printing products (e.g. for stages) had to be seamed together. This procedure not only caused additional costs, but also quality issues such as color differences and mismatches. Secondly, the fabrics are generally delivered in a coiled and nonaligned state. The result is that end and shoot are not aligned vertically to each other. Hence, the printed image would have shown distortions. Especially, in theatre such flaws pose an unacceptable visual 
irritation. Hence, BI had to apply an additional alignment process for each delivered fabric roll, which required a high degree of manual work.

Thus, an endless printing technology had to be developed, where twelve meter wide materials in fabric grain direction are prepared and introduced into the printing system. The challenge on behalf of MSD was the development and construction of an efficient and accurate technology for the actuation and material guiding of the printing system. Within this project, the FAD as a separate machine states a subproject, which is integrated in „Infinitus“. The device had to prepare the fabric by storing it in a depot and introducing it to the printing system.

The case study can be considered as a typical approach of the design of technical systems and products (cf. Pahl et al., 2013). The development of the FAD was characterized by the coarse phases of clarifying and defining the task, making a concept, designing and elaborating the solution, and finally the realization of the product. The development team had to face various problems and knowledge gaps. Overall problems, which had to be solved, were the preparation of the materials for the later printing system, i.e. the alignment of textiles in fabric grain direction, the preservation of the achieved shape, and the preparation of the materials in a way to feed the following printing process. At the beginning, requirements for the overall project were collected. Based on the functional structures of the system, solutions of the sub-functions were developed. The treated fabrics had to be fastened and held at its edges as well as the materials were supposed to be transported evenly and exactly during the whole process. The current orientation of the cords needed to be gathered and deviations from target alignment had to be calculated. Through an appropriate actuation system, the fabrics had to be aligned purposefully and the quality of the materials needed to be checked during or after the preparation. Another challenge was the development of an appropriate control system to drive the previous mentioned modules. After drawing up possible subsolutions for the different sub-functions, they were analyzed and rated with the help of research, calculations and material tests. Here, insights of textile behavior were created by pulling and friction tests. Additionally, a special test rig was developed to evaluate an actuator-roller solution principle, where the fabrics on both sides were compressed together through rollers, arranged in a line and separately driven. Calculations, simulations, and computer aided design (CAD) models supported the investigations. Finally, the device was realized via manufacturing, mounting and an implementation of the control system.

The final FAD (Figure 1) consists out of the following main components: 1) the welded frameworks (upper and lower); 2) a hoisting device; 3 ) the fixings of the lateral edges to strain and fasten the edges during machining using spiked chains; 4) the alignment of the lateral edges by a grabber; 5) a camera system to monitor the orientation of the materials fiber; 6) the actuator system - composed out of 13 one meter wide actuator rollers, separately driven by electric motors and gears - to strain and align fabrics regarding to the determined orientation of cords; 7) the fixing to hold the material over the full width; 8) a humidifier and a drying plant for conserving the target state of the fabric before printing.

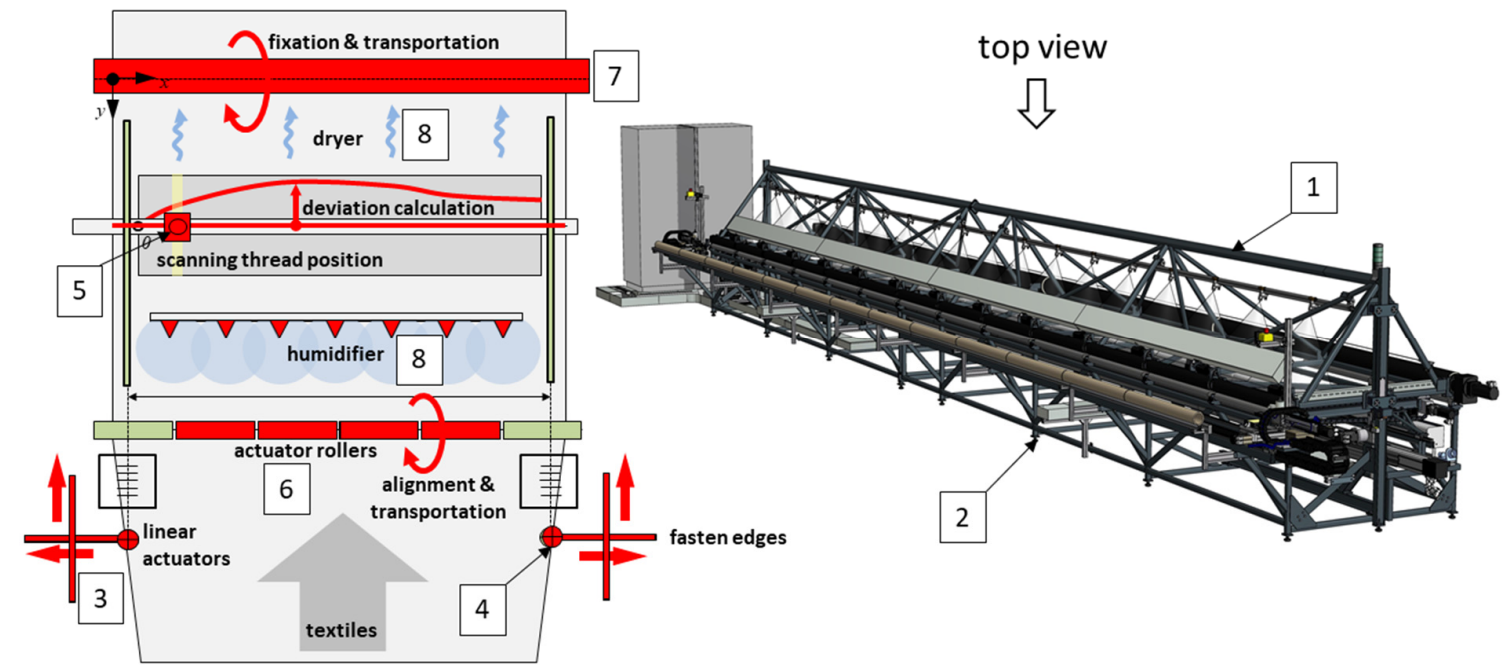

Figure 1. Operating mode and CAD-model of the fabric alignment device 
The resulting device is able to align coiled or folded fabrics from three up to 12.5 meters width in fabric grain direction with a speed up to 100 square meter per hour. The delivered materials can be converted and stored in a depot to meet the required mechanical properties for the „endless“-printing system process. After completion at MSD, the device was dismounted, packaged and transported to BI, where it was rebuilt, integrated and tested.

\subsection{Case study 2: Bioinspired materials}

The case study "Bioinspired Materials" refers to the project "Hierarchy of microstructural features as the origin of fracture resistance in dentine and ceramic composites". It was funded from 2009 to 2015 by the German Research Foundation (DFG) within the priority program 1420 "Biomimetic Materials Research: Functionality by Hierarchical Structuring of Materials", where researchers from CharitéUniversitätsmedizin Berlin (Dental materials and Biomaterial Research), Max Planck Institute for Colloids and Interfaces (Dept. Biomaterials), and TU Berlin (Chair of Materials Engineering) worked together. The team consisted mainly of materials scientists, but also included chemists and dentists. The researchers collaborated in several sub-projects, such as mechanical characterization of the whole teeth, mechanical and microstructural investigation of dentine, manufacturing of ceramic materials and, mechanical and microstructural analysis of the ceramic. Findings and insights obtained by a single researcher or a team of researchers of a sub-project, as well as technical or scientific problems, were regularly communicated in meetings. These meetings and additional discussions were often used to solve problems jointly in the group. Experiments were often planned and carried out together, too.

The aim of the project was, on the one hand, to investigate functional structures and principles in biological materials. On the other hand, this understanding of the structure-function-relationships on different length-scales should be used to create novel, resilient synthetic materials with outstanding property combinations as zirconia ceramic. Here, teeth are promising study objects, as they are mostly long lasting structural elements of moderate maintenance needs. Dentine - the interior and the main component of the tooth - is a material of the bone family and has a corresponding hierarchical structure. At the nano level, it is composed of a fibrous protein matrix (including $90 \%$ collagen I), which is mineralized by calcium hydroxyapatite and water (Weiner and Wagner, 1998). Unlike bone, dentine is not capable to heal by remodeling. Therefore, it is particularly interesting to investigate the damage tolerance of dentine and the influence of the structural elements on it. On a higher level, dentine contains micro sized features, for example dentinal channels (tubules), and graded structures. The interaction of the structural components is very complex, which so far has not been fully understood.

Zirconia ceramic is used as dental material due to its high hardness and a good aesthetic appearance. This ceramic reveals a higher toughness than other ceramics, but is still brittle. Zirconia occurs as a polymorph in cubic, tetragonal and monoclinic crystal structure, where the atoms are arranged in the correspondent pattern. The toughness of certain zirconia compositions is based on a process of transformation toughening where the tetragonal structure transforms into the monoclinic structure by mechanical induction: if a crack hits the tetragonal structure, it will convert into the monoclinic structure. The monoclinic structure is less dense and thus, a larger volume arises locally in the material. As a result, a local compressive stress field is created that arrests or slows down crack propagation. Thus, the toughness of the material increases, which results in a higher fracture resistance (Piconi and Maccauro, 1999).

At the beginning of the project, it was important to understand where and why cracks in dentine appear, propagate and stop. It has to be noted, that the studies on dentine were continued throughout the project. Furthermore, it was needed to know more about the extent of the transformations zone in zirconia. Firstly, monolithic zirconia was analysed, which is used in dental laboratories. Both, dentine and zirconia were investigated by combined mechanical testing with advanced materials characterization methods. The research team gained important insights, for example, about the crack paths in dentine as well as the structure-related relationships between the nano-sized components.

Secondly and parallel to these first investigations and results, the researchers developed a manufacturing process of a structured ceramic. It allowed to create micro sized features and graded structures like those identified to be important for the fracture resistance of dentine. Findings from the partners studying the natural material were incorporated into the structured ceramics, which, in turn, made developments on 
the manufacturing side necessary. The collaborated scientists experimented with different common ceramic technologies as slip casting and powder compaction with some success. However, it was obvious that the development of structured and, specifically, of graded ceramics requires its own technological development. Hence, the activities focused on an additive manufacturing method where a ceramic body is formed layer by layer - the electrophoretic deposition process (EPD, Figure 2). The manufacturing method was developed to make layers of different thickness, composition or, porosity Additionally, mechanical tests were used to validate the modelling of the mechanical behavior of natural and synthetic materials in-silico in order to better understand the complex fracture behavior in dentine as well as in ceramics.

a)

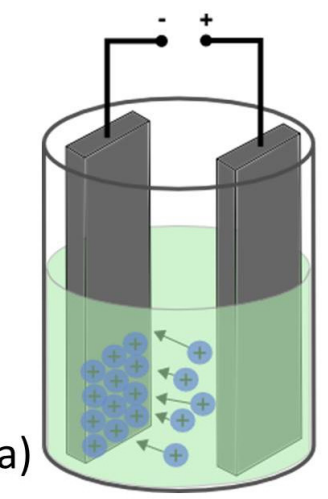

b)

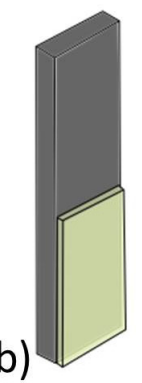

c)

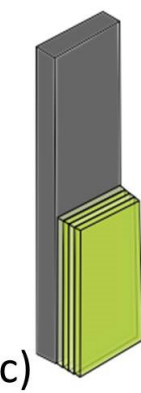

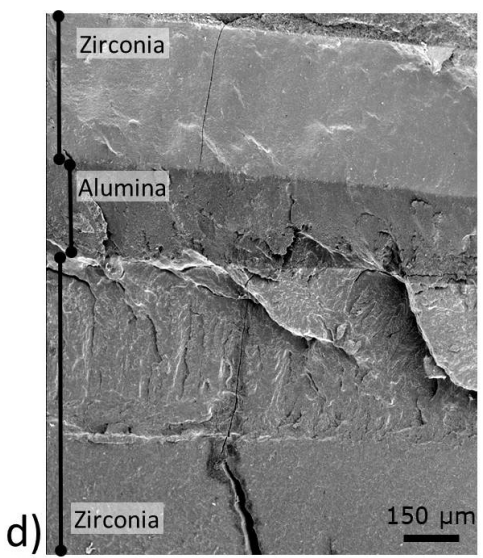

Figure 2. a) Schematic representation of the electrophoretic deposition process showing attraction of positively charged ceramic particles towards the anode; b) single layer deposition; c) deposition of multilayers from two different zirconia suspensions; d) fracture surface of a simple layered ceramic construct depicts layers of different compositions and thicknesses

The third phase of the project "bioinspired materials" was the analysis of the new self-developed and self-manufactured ceramic. Therefore, the ceramic constructs were transferred between the manufacturing and characterization teams. Significant new insights into the extension of the transformation zone and its impact on crack propagation in structured ceramics were gained by nano indentation, various X-ray analyses and combined methods (Mochales et al., 2011; Maerten et al., 2013; Zehbe et al., 2016). These crucial findings were once again exploited by designing improved structured ceramics and their manufacturing. This can be described as the fourth phase of the project, which is still going on.

\section{Application of the conceptual framework on the case studies}

In order to evaluate the applicability of the conceptual framework, we will analyse activities of both case studies and apply them to the core concepts in the following.

\subsection{Knowledge acquisition}

Case study 1: One example for the development of the FAD was collecting, merging and processing of knowledge in order to generate the list of requirements for the endless printing machine as well as for the FAD and its assemblies. This list describes demands, desires and information on the product both in quantitative and qualitative terms. Thus, it plays a fundamental role for later steps in the product development process, while some requirements can still change or may be added. The requirements of the product, e.g. the minimal printing size of twelve meters, the implementation of existing textile rollers as part of the new system, or the prevention of residues on the fabric during processing, were gathered. This process took place by research, by interviews with the customer BI (in order to receive special 
product expertise such as interface definitions with regard to the material supply of the printing system), and by internal discussions of the MSD-team (e.g. in order to define requirements for safety facilities). Case study 2: For the development of bioinspired materials, it was essential to acquire the state of the art in the biological as well as the technical system. The basic knowledge about teeth and dentine regarding both structure and mechanical behavior - was collected from standard dental medicine books (cf. Nanci, 2007) and scientific publications as well as by communications with the dentists. The procedure was similar for zirconia. Publications and standard works were used in order to merge existing knowledge about mechanical - especially toughness - behavior applied to dental ceramics.

\subsection{Knowledge transformation}

Case study 1: In the sub-project of the FAD, a solution for the sub-function "alignment in fabric grain direction" had to be found. Research revealed a common application of rollers in the field of textile industry to transport fabrics. This principle was transformed into the context of systematic straining. 13 rollers, used as actuators, were arranged in series and they could be triggered individually to enable a targeted alignment of the fabrics. Thus, it presented a modified application of already existing textile rollers.

Case study 2: The material-scientific approach on dentine as a nanocomposite represents knowledge transformation since neither biological, chemical nor physical, but mechanical interactions between the components and their effects on the behavior of the material were investigated. Different physical methods such as X-ray diffraction (XRD) and other scattering methods were transformed to investigate the origin of fracture resistance in dentine due to their adaptation and modification for a humid environment, which is required for in-situ mechanical testing of biological materials. Furthermore, the array of mechanical tests included tensile, bending and compression loading combined with various 2D and 3D characterization techniques comprising advanced optical, electron and X-ray microscopy. The responses to load and interactions with microstructure were tracked spanning the length-scale from single natural (apatite) and synthetic (alumina/zirconia) crystals to full-field surface and bulk deformation-patterns. For this range of observed length scale, the adaptation and extension of the experiments were necessary.

\subsection{Knowledge generation}

Case study 1: The development of the FAD used tests to learn more about the textile's behavior. Those were necessary to realize and to evaluate possible technical solutions. Knowledge about the textile's static and dynamic coefficient of friction on stainless steel or aluminum, distortion characteristics and related tensile forces was created with the help of diverse tests. They included friction tests, manual pulling in warp and weft thread direction as well as an alignment test rig, which consisted out of two separately driven rollers. For instance, the latter revealed the required motor torque for the actuator roller drive and an assessment of this sub-solution principle to align materials in fabric grain direction.

Case study 2: New findings by applying combined analysis methods to the investigation of toughening mechanisms in dentine in terms of the present compressive residual stress of the mineral phase (Forien et al., 2015) gave a new impact for the development of synthetic materials. In addition, detailed knowledge has been generated by technology-based tools, such as simulation and modelling of biomechanics and new preparation techniques. In the fundamental research, the team investigated for instance the lateral expansion of the tetragonal-monoclinic transformation in order to better understand this process. These new insights about the effects of structural features on the local distribution of the transformation contributed to create a new manufacturing process of hierarchically structured ceramics for improved dental applications.

\subsection{Knowledge integration}

Case study 1: One example is the integration of the device into the overall "Infinitus"-project. Since the beginning of the product, development process of the device was coordinated by defined interfaces. The FAD with its functioning characteristics was merged with other assemblies, e.g. the printing system, in order to synthesize a new product. 
Case study 2: In the course of the development of the electrophoretic deposition layering process, different findings about the variety of effects of structural features in dentine and zirconia determined in the work groups had to be merged to optimize the complex process and to manufacture layered ceramics with hierarchical structure, different composition and thickness. Furthermore, knowledge on analysis methods, so far mainly used separately, had to be united by combining characterisation methods in a new manner (for example XRD in humid environment combined with in-situ mechanical tests). The integration of knowledge is an important aspect of knowledge dynamics because the related activities unite knowledge of different work groups. This was achieved by comprehensive essays (Zaslansky et al., 2016) as well as by reports to the DFG and new applications.

\subsection{Knowledge transfer}

Case study 1: In the "Infinitus"-project knowledge, e.g. progresses, was shared between the working group of MSD and the customer BI as well as in the corresponding teams. Knowledge was transferred in interviews between the research group and the customer, in discussions and in meetings within the, as well as by using databases (e.g. dropbox). Furthermore, the FAD itself, as a manifestation of knowledge, was transferred from the workshop at TU Berlin, where it was constructed and built, to the premises of BI.

Case study 2: On the one hand, knowledge (meetings, discussions and problems solving) and materials (transferred from team to team) circulated within the project. On the other hand, the transformed and generated knowledge of the bioinspired-materials-project was passed on to the scientific community by publishing, more precisely, by writing peer-reviewed articles or by presenting results at international conferences (see also Section 4.6 "Knowledge manifestations"). Interestingly, the transfer of knowledge occurred not only within materials science, but also to the corresponding journals in physics, chemistry, and dentistry.

\subsection{Knowledge manifestations}

Case study 1: An example of knowledge manifestation is the physical form of the alignment construction after mounting, i.e. device as an artefact. Knowledge manifests integrated in the overall construction as a functioning machine for printing fabric materials. The existence of the device decouples the applied knowledge from individuals. By analysis, the embodied knowledge of the device could be made accessible again. Further manifestations of the project were CAD-models, technical drawings as well as project reports.

Case study 2: New knowledge manifests in material science by publications in scientific journals, patents and other publications. The output of six years was more than 20 publications in peer-reviewed papers, two patents and more than 20 lectures at international conferences. Furthermore, instructions for additive manufacturing of structured ceramics were gained.

\section{Discussion}

The case studies in our investigation serve as a "test environment" to further explore the conceptual framework. As the cases of the FAD and of bioinspired materials can be regarded as opposing poles of an imaginary spectrum of instances of knowledge dynamics in engineering science - the one being primarily application-oriented research, which aims to develop a unique product, the other being primarily fundamental research, which aims to explore generalizable insights and laws -, they constitute helpful tools to test, refine and revise our heuristic of core concepts. The exemplary application of the conceptual frameworks on both case studies shows that the analysis can be used to identify and capture activities of knowledge processing. Instances from the case studies exemplify the knowledge terms, and hence, allow to render the characterizations of the terms plausible. As it is, the examples only capture snapshots of very complex processes. Whether the framework is capable of describing a complete development or research project needs still to be investigated.

However, what can be pointed out already right now: the knowledge related activities captured by the terms, can be found in many phases and at different levels. Hence, the application of the concept allows 
to identify several perspectives to which the different knowledge terms can be applied (Figure 3), such as

- level of observation, i.e. the degree of detail in which an engineering-scientific project is to be considered (e.g. macro, meso and micro);

- the object of investigation, i.e. the object that sets the frame for the investigation (e.g. a productoriented approach or a project-oriented approach);

- time, i.e. specific periods of time during which certain activities are carried out in the context of engineering-scientific projects (e.g. phases of a product development or a research project).

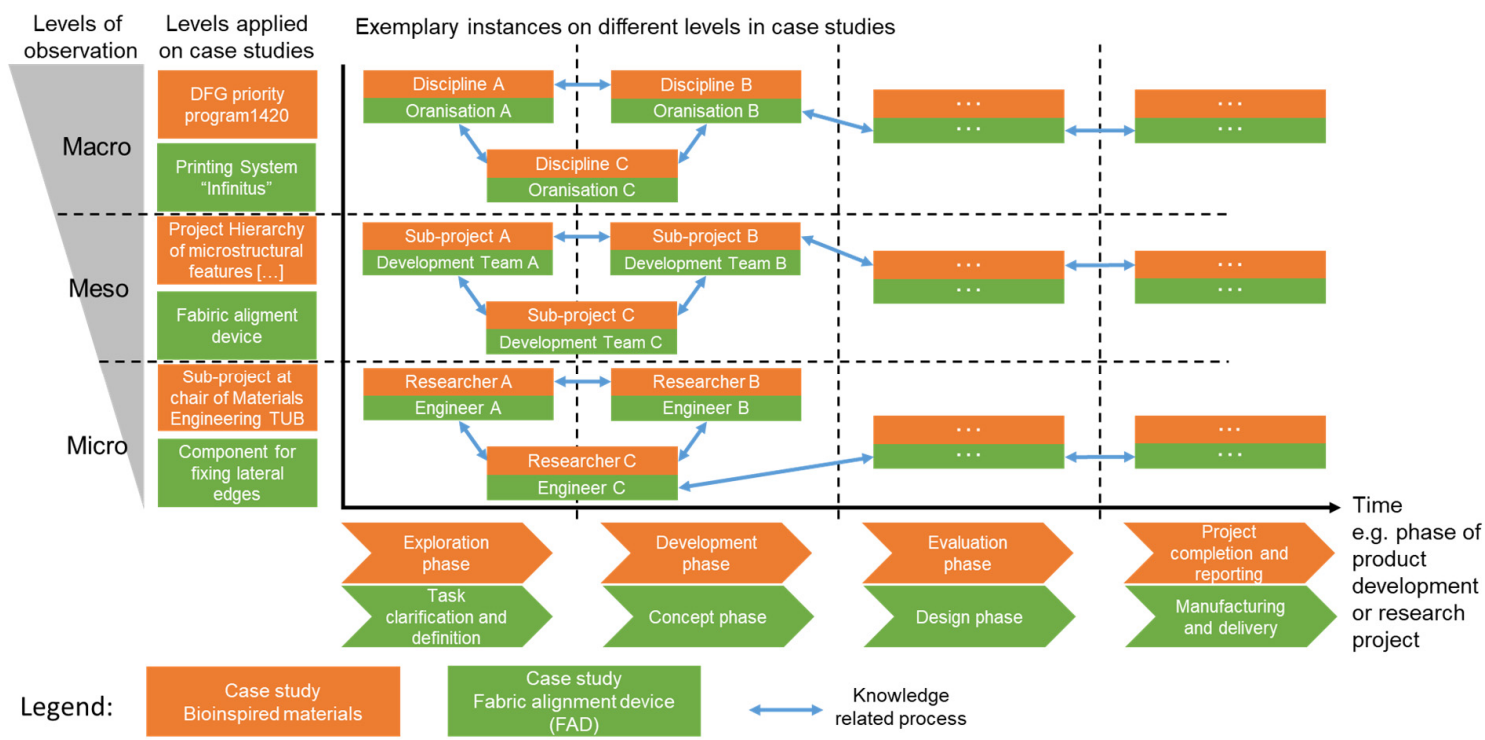

Figure 3. Proposed perspectives of the conceptual framework exemplarily applied on the case studies

We want to illustrate the differing perspectives by drawing on examples from the case studies. Let us start with the level of observation by using the "bioinspired materials" case study. The DFG priority program 1420 represents the macro level in which the project "bioinspired materials research" could be investigated. If the framework is applied at this level, the focus will be on the interaction of the disciplines involved. In this case, the exchange of knowledge between the disciplines during the course of the project is of major interest. This level of observation can be used, for example, to investigate larger connections between disciplines in an historical context. The research project "Hierarchy of microstructural features as the origin of fracture resistance in dentine and ceramic composites" represents a meso level with a certain subset of disciplines, which were funded for a specific research question within this particular network. At this level, the knowledge processes between sub-projects would be of major interest. Thus, the focus could be on persons and the composition of teams and their respective affiliation to a certain institution. The micro level in this example with a high degree of detail would be the individual sub-project, which was located at the chair of materials engineering. In this context, the knowledge processes that exist between individuals within the sub-project will be in the focus of the investigation.

In the latter case, the analysis was coupled with a project-oriented approach as the object of investigation. What happens, if we take the product as the object of investigation? We want to illustrate this perspective of analysis by using the case study of the fabric alignment device. Its development can be divided into the following levels: the overall printing system as product level (macro level), the FAD as sub-product level (meso level), the actuator system as an assembly level (also meso level) and one roller drive as a component level (micro level). Within this setting, examples can be found in case of the product development phase „clarify and define the task“. On the product level, the overall list of requirements can be stated. Analogously, for the sub-product level, the requirements for the discussed 
device can be stated according to defined interfaces, just as for the assembly level. Lastly, there is a needed driving torque for the roller drive (component level) derived from the determined textile behavior. The product development can be coarsely divided into: a concept phase in which the requirements and the tasks are clarified and the further process is planned; a design phase in which the product is actually designed; a validation phase in which the designed product is validated against given requirements, whereby design and validation phase can undergo several iterations if the design fails to meet the requirements; and the final phase of manufacturing in which the designed product is physically realized. Finally yet importantly, the framework allows to highlight the perspective of time within an analysis. It can be applied to different time scales, e.g. the investigation of a certain phase of product development or a research activity, the overall process of development of an artefact, as well as long-term studies of the development of technical objects or disciplinary transformations. An example for the latter comes with the "bioinspired-materials-project". The project drew on bodies of knowledge, which were transformed from science into materials sciences decades before: Zirconia is known in its natural type as a mineral (baddeleyite). The polymorphism and related different volumes were discovered in natural science by Xray diffraction methods at the beginning of the 20th century (Ruff and Ebert, 1929). However, the volume effects were not applied for a long time. Since the 1950s, the material has been investigated by materials scientists and other engineers (Duwez et al., 1951; Bailey, 1964). The knowledge generated in natural science was transformed in the last decades of the 20th century, and only by then it gained technical relevance by using the transformation toughening mechanism described above (Garvie, 1975).

\section{Conclusion and outlook}

The conceptual framework, albeit not a process model in itself, allows for shedding light on important aspects of dynamics of knowledge in engineering science. As discussed before, the presented conceptual framework can be used as an analytical approach to better understand the dynamic behavior of knowledge and knowledge processing in engineering science. In the current approach, the analysis is therefore more retrospectively oriented. As the framework is still in development, subject to further research will be the question whether the key concepts are sufficiently selective and whether they need to be elaborated in more detail.

However, there are various ways in which the framework can be used to support knowledge processing in both scientific and practical terms. In scientific terms, a better understanding of the mechanisms of exchange in research networks can help to improve the efficiency of knowledge exchange and to uncover new ways to link knowledge resources. For industrial practice, an in-depth understanding of how knowledge is created, circulated and applied within the company can help to increase innovation and product quality. By applying the proposed framework, previously hidden knowledge gaps can be identified and thus errors in product development might be avoided at an early stage. In product development, such gaps occur primarily at the intersections between individual trades (e.g. between components or assemblies) or disciplines (e.g. between electronics and mechanics), where different domains of knowledge meet with their respective models and tools (Stöckert, 2010). Regarding innovation capability, the proposed framework can be used to identify central stakeholders whose specific knowledge have significant influence on innovation processes. This allows to introduce new impulses, for example, in order to intentionally expand possible solution spaces (McKilligan et al., 2017). Whether and to what extent the framework can be used to support knowledge processing in science and industry in a prospective way still needs to be further researched.

Finally, we only used the framework as a descriptional tool in order to better understand knowledge production and distribution in engineering science so far, but also to better understand the characteristics of engineering science as an academic discipline (in contrast, e. g. to the natural sciences, social sciences and humanities). However, the framework also lays the basis for a normative application of the insights gained through the investigation of the case studies by developing recommendations how to (better) deal with knowledge-related phenomena in engineering sciences.

\section{Acknowledgements}

This research received support from the German Research Foundation (DFG, Grant Agreements no. AM 405/4-1, STA 1112/15-1, ME 2046/8-1). 


\section{References}

Bailey, J.E. (1964), “The Monoclinic-Tetragonal Transformation and Associated Twinning in Thin Films of Zirconia", Proceedings of the Royal Society of London. Series A, Mathematical and Physical Sciences, Vol. 279 No. 1378, pp. 395-412. http://www.jstor.org/stable/2414822

Duwez, P., Brown Jr., F.H. and Odell, F. (1951), "The Zirconia-Yttria System”, Journal of The Electrochemical Society, Vol. 98 No. 9, pp. 356-362. https://doi.org/10.1149/1.2778219

Forien, J.-B., Fleck, C., Cloetens, P., Duda, G., Fratzl, P. et al. (2015), “Compressive Residual Strains in Mineral Nanoparticles as a Possible Origin of Enhanced Crack Resistance in Human Tooth Dentin”, Nano letters, Vol. 15 No. 6, pp. 3729-3734. https://doi.org/10.1021/acs.nanolett.5b00143

Garvie, R.C. (1975), “Ceramic steel?”, Nature, Vol. 258, pp. 703-704. https://doi.org/10.1038/258703a0

Gausemeier, J. and Wiendahl, H.-P. (2011), "Hebel zur Gestaltung von Produktentstehung, Produktion und Wertschöpfung in Deutschland - Zusammenfassung und Schlussfolgerungen”, In: Gausemeier, J. and Wiendahl, H.-P. (Eds.), Wertschöpfung und Beschäftigung in Deutschland, Springer Berlin Heidelberg, Berlin, Heidelberg, pp. 103-108. https://doi.org/10.1007/978-3-642-20204-9_7

Lünnemann, P., Müller, P., Neumeyer, S., Wang, W.M., Hayka, H. and Kirsch, L. (2016), Zukunft der unternehmensübergreifenden Kollaboration: Expertenmeinungen zu aktuellen Herausforderungen und zukunftsweisenden Trends in der kollaborativen Produktentwicklung, 1st ed., Fraunhofer IPK, Berlin.

Maerten, A., Zaslansky, P., Mochales, C., Traykova, T., Mueller, W.D. et al. (2013), "Characterizing the transformation near indents and cracks in clinically used dental yttria-stabilized zirconium oxide constructs", Dental materials, Vol. 29 No. 2, pp. 241-251. https://doi.org/10.1016/j.dental.2012.10.008

McKilligan, S., Jablokow, K.W., Daly, S.M. and Silk, E. (2017), "Usability tests of ideation flexibility tools with engineering design practitioners", CoDesign, Vol. 80 No. 7, pp. 1-21. https://doi.org/10.1080/15710882.2017.1325909

Mochales, C., Maerten, A., Rack, A., Cloetens, P., Mueller, W.D. et al. (2011), "Monoclinic phase transformations of zirconia-based dental prostheses, induced by clinically practised surface manipulations", Acta biomaterialia, Vol. 7 No. 7, pp. 2994-3002. https://doi.org/10.1016/j.actbio.2011.04.007

Nanci, A. (2007), Ten cate's oral histology-pageburst on vitalsource: development, structure, and function, Elsevier Health Sciences.

Neumann, F. (2015), Analyzing and Modeling Interdisciplinary Product Development, Springer Fachmedien Wiesbaden, Wiesbaden. https://doi.org/0.1007/978-3-658-11092-5

North, K. (2011), Wissensorientierte Unternehmensführung. Wertschöpfung durch Wissen, 5th ed., Gabler Verlag / Springer Fachmedien Wiesbaden $\mathrm{GmbH}$, Wiesbaden.

Pahl, G., Beitz, W., Schulz, H. and Jarecki, U. (2013), Pahl/Beitz Konstruktionslehre: Grundlagen erfolgreicher Produktentwicklung. Methoden und Anwendung, Springer-Verlag, Berlin Heidelberg. https://doi.org/10.1007/978-3-642-29569-0

Piconi, C. and Maccauro, G. (1999), “Zirconia as a ceramic biomaterial”, Biomaterials, Vol. 20 No. 1, pp. 1-25. https://doi.org/10.1016/S0142-9612(98)00010-6

Ruff, O. and Ebert, F. (1929), "Beiträge zur Keramik hochfeuerfester Stoffe. 1. Die Formen des Zirkondioxyds", Zeitschrift für anorganische und allgemeine Chemie, Vol. 180 No. 1, pp. 19-41. https://doi.org/10.1002/zaac.19291800104

Stöckert, H. (2010), Fehlervermeidung an Schnittstellenprozessen der verteilten Produktentwicklung, PhD thesis, Technische Universität Berlin.

VDI (2008), VDI Richtlinie 5610 - Wissensmanagement im Engineering - Grundlagen, Konzepte, Vorgehen, Beuth Verlag, Berlin.

Verhagen, W.J.C., Bermell-Garcia, P., van Dijk, R.E.C. and Curran, R. (2012), “A critical review of KnowledgeBased Engineering. An identification of research challenges", Advanced Engineering Informatics, Vol. 26 No. 1, pp. 5-15. https://doi.org/10.1016/j.aei.2011.06.004

von Kutschera, F. (1982), Grundfragen der Erkenntnistheorie, Walter de Gruyter, Berlin. http://doi.org/10.1515/9783110841251

Wang, W.M., Göpfert, T. and Stark, R. (2016), "Data Management in Collaborative Interdisciplinary Research Projects-Conclusions from the Digitalization of Research in Sustainable Manufacturing", ISPRS International Journal of Geo-Information, Vol. 5 No. 4, p. 41. https://doi.org/10.3390/ijgi5040041

Weiner, S. and Wagner, H.D. (1998), "THE MATERIAL BONE: Structure-Mechanical Function Relations", Annual Review of Materials Science, Vol. 28 No. 1, pp. 271-298. https://doi.org/10.1146/annurev.matsci.28.1.271

Zaslansky, P., Currey, J.D. and Fleck, C. (2016), "Learning from evolutionary optimisation: what are toughening mechanisms good for in dentine, a nonrepairing bone tissue?", Bioinspiration \& biomimetics, Vol. 11 No. 5 , p. 51003. https://doi.org/10.1088/1748-3190/11/5/051003 
Zehbe, R., Mochales, C., Radzik, D., Müller, W.-D. and Fleck, C. (2016), "Electrophoretic deposition of multilayered (cubic and tetragonal stabilized) zirconia ceramics for adapted crack deflection", Journal of the European Ceramic Society, Vol. 36 No. 2, pp. 357-364. https://doi.org/10.1016/j.jeurceramsoc.2015.08.022

Wei Min Wang, Researcher Technische Universität Berlin, Industrial Information Technology Pascalstr- 8-9, 10587 Berlin, Germany

Email: w.wang@tu-berlin.de 